\section{NUCLEAR} INSTRUMENTS

8. METHODS

IN PHYSICS

RESEARCH

ELSEVIER

\title{
Light charged particle and intermediate mass fragment cross-sections in $\mathrm{GeV}$ proton-induced reactions
}

\author{
V. Bollini ${ }^{\mathrm{a}}$, A. Bubak ${ }^{\mathrm{b}}$, A. Budzanowskic, J. Cugnon ${ }^{\mathrm{d}}$, D. Filges ${ }^{\mathrm{a}}$, F. Goldenbaum ${ }^{\mathrm{a}, *}$, \\ A. Heczko ${ }^{\mathrm{e}}$, H. Hodde ${ }^{\mathrm{f}}$, L. Jarczyk ${ }^{\mathrm{e}}$, B. Kamys ${ }^{\mathrm{e}}$, M. Kistryn ${ }^{\mathrm{c}}$, St. Kistryn ${ }^{\mathrm{e}}$, \\ St. Kliczewski ${ }^{\mathrm{c}}$, A. Kowalczyk ${ }^{\mathrm{e}}$, P. Kulessa ${ }^{\mathrm{a}, \mathrm{c}}$, H. Machner ${ }^{\mathrm{a}}$, A. Magiera ${ }^{\mathrm{e}}$, W. Migdal ${ }^{\mathrm{e}}$,

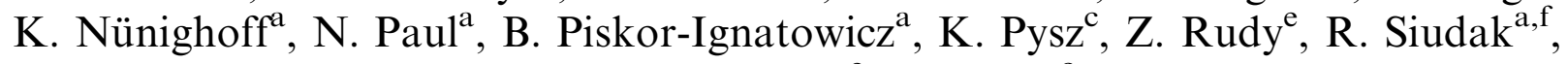 \\ M. Wojciechowski ${ }^{\mathrm{e}}$, E. Kozik ${ }^{\mathrm{c}}$ \\ ${ }^{a}$ Institut für Kernphysik, Forschungszentrum Jülich GmbH, Germany \\ ${ }^{\mathrm{b}}$ Univ. Silesia, Katowice, Poland \\ ${ }^{\mathrm{c}}$ Institute of Nuclear Physics, INP-PAN Krakow, Poland \\ ${ }^{\mathrm{d}}$ Univ.de Liege, Belgium \\ ${ }^{\mathrm{e}}$ Jagellonian Univ. Krakow, Poland \\ ${ }^{\mathrm{f}}$ Univ. Bonn, Germany
}

Available online 3 March 2006

\begin{abstract}
As an international collaboration PISA (Proton Induced SpAllation) has initiated measurements of total and double differential crosssections for products of spallation reactions at the proton accelerator COSY in Jülich (Germany) in order to study secondary particle production created in structural, window, and target materials by proton beams up to $2.5 \mathrm{GeV}$ incident kinetic energy. In the framework of Spallation Neutron Sources and accelerator-driven systems, residual nuclei ( $\mathrm{H}$, He up to intermediate mass fragrments) production cross-sections are of great importance for estimation of damage in target and structural materials. Reliable and comprehensive experimental data, especially for p-energies beyond $1 \mathrm{GeV}$ will serve as benchmarks for code development and validation. Data taken by Bragg curve spectroscopy and silicon detector telescopes for the reaction $1.9 \mathrm{GeV} \mathrm{p}+\mathrm{Ni}(\mathrm{Au})$ will be discussed and kinetic energy spectra of light ions and intermediate mass fragments will be presented.
\end{abstract}

(C) 2006 Elsevier B.V. All rights reserved.

PACS: 25.40.S; 21.60; 13.75.-n

Keywords: Spallation; Fragmentation; Nuclear reaction; Double differential cross-sections; Composite particles; Energy spectra

\section{Motivation}

The Proton Induced SpAllation (PISA) project aims at the measurement of total and double differential crosssections for products of spallation reactions on a various targets $(\mathrm{C}-\mathrm{Au})$, induced by protons of energies between 100 and $2500 \mathrm{MeV}$. Measurements of cross-sections and the investigation of the interaction of medium and high energy protons with atomic nuclei are important for

\footnotetext{
*Corresponding author. Tel.: + 492461614250 .

E-mail address: f.goldenbaum@fz-juelich.de (F. Goldenbaum).
}

providing benchmark data in the GeV incident p-energy range [1-3], understanding the complex reaction mechanism itself [3] and testing the reliability of physical models [2] describing the fast intranuclear cascade (INC) phase as well as the subsequent statistical decay from an equilibrated or thermalized hot nucleus. A particular focus is on developing new models for the description of highly energetic composite particles. These investigations play a major role in planning and construction of high intensity spallation neutron sources [4-8], since the production cross-sections are of particular interest for studying radiation damage in target, window and structural materials. In the astrophy- 
sical context $\mathrm{Li}, \mathrm{Be}$ and $\mathrm{B}$ data in proton-induced reactions on light targets (up to $\mathrm{Fe}$ ) are of crucial importance $[9,10]$. As concerns the dynamics of the nuclear reactions, many questions are by far not yet fully understood: the approach to thermal equilibrium [3], competition between sequential and simultaneous emission of fragments [11,12], the production mechanism of intermediate mass fragments (IMF) and its relation to possible liquid-gas phase transition [13], expansion of the nucleus during excitation and subsequent decay.

The dependence of the production cross-sections as a function of bombarding energy and mass of the decaying system should shed light on the competition of various mechanisms of interaction of protons with nuclei. Therefore one of the main tasks of the PISA investigations is the determination of various IMF yields in their full kinetic energy range for several targets and incident proton energies.

\section{Experimental set-up}

The PISA experiment has eight detection arms arranged around a scattering chamber. Currently, only the most forward $\left(15^{\circ}\right)$ and the most backward $\left(120^{\circ}\right)$ arms with respect to proton beam direction are mounted. Each detection arm consists of two multichannel plates (MCP) working as "Start" and "Stop" detectors for the time of flight measurement, a Bragg Curve Detector (BCD) $[14,15]$ followed by three silicon detectors of 100, 300 and $4900 \mu \mathrm{m}$ thickness for particle identification using $\Delta E-E$ techniques and kinetic energy measurement of intermediate-mass spallation products, and a set of double-layer scintillation detectors - fast and slow (phoswich) - in order to identify light-charged evaporation and spallation products like $\mathrm{p}, \mathrm{d}$, $\mathrm{t}$, He. Time-of-Flight plus BCDs provide identification of light heavy ions with mass up to 20-30 and kinetic energy starting from less than $1 \mathrm{MeV} / \mathrm{amu}$.

\subsection{The Bragg curve detector}

Bragg curve spectroscopy is used to identify highly ionizing particles. BCDs detect fragments with high precision over a broad range of nuclear charges with low registration thresholds [16-21]. The BCD-design employed here, and results of the PISA test experiment are presented in Refs. [14,15,22,23,24]. For charged, nonrelativistic particles the Bethe-Bloch formula for specific energy losses in a given medium can be simplified to: $-\mathrm{d} E / \mathrm{d} x \sim c Z^{2} / E$, where $Z, E$ are the atomic number and kinetic energy of the detected particle and $c$ contains all relevant constants together with the quantities characterizing the detector medium. Isotope separation for detected particles is achieved for elements up to nitrogen when, in addition to the usual charge identification by Bragg spectroscopy in the PISA experiment, TOF information is taken into account. If the particle stops in the active chamber volume, apart from the measured TOF, the following values are calculated from the pulse shape: the integral of the specific ionization over the track (total kinetic energy $E$ of the particle), the maximum of the Bragg Peak (BP) from the maximum of the specific ionization of the ion (BP proportional to $Z$ ), the duration $R$ (corresponding to the range in the BCD gas volume) and a partial integral from the specific ionization at the beginning of the track $(\sim \Delta E / \mathrm{d} x)$. Isotope identification is performed by correlations between the parameters $R, E, \Delta E$ and TOF.

\section{Experimental results of PISA}

At the internal beam of COSY the investigation of the reactions induced by protons on thin targets $\left(50-200 \mu \mathrm{g} / \mathrm{cm}^{2}\right)$ enables us to get the cross-sections without the absorption and energy loss involved with the propagation of reaction products in the material of the target. The multiple circulation of the beam in the COSY ring is used to compensate for the small reaction rate of beam-protons with the thin targets.

\subsection{Particle identification using $B C D$}

We observe in the BCDs the unambiguously identified charge of fragments from helium up to silicon, i.e. $2 \leqslant Z \leqslant 14$. Fig. 1 illustrates this, showing the identification spectrum (Bragg curve peak BP versus energy $E$ deposited in the gas volume) for the reaction $1.9 \mathrm{GeV} \mathrm{p}+\mathrm{Ni}$ at fragment emission angles of $15^{\circ}$ with respect to the proton beam. The distinction of the charge in range $2 \leqslant Z \leqslant 14$ (for $15^{\circ}$ ) is visible. Most energetic particles with $Z \leqslant 6$ (for $15^{\circ}$ ) have a range larger than the length of the $\mathrm{BCD}$. The resolution of charge distribution $\Delta Z$ has found to be between $10 \%$ and $12 \%$ per amu. After selecting an element of given $Z$ in the Bragg-curve identification spectrum shown in Fig. 1, an isotope separation or mass identification of the emitted fragments is possible due to different time-

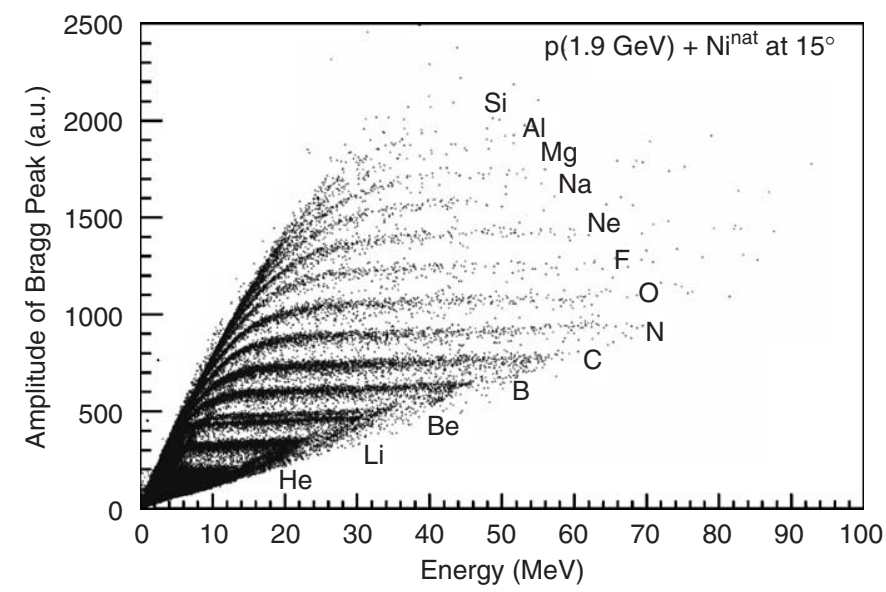

Fig. 1. Identification spectrum of emitted fragments in forward $\left(15^{\circ}\right)$ direction following $1.9 \mathrm{GeV} \mathrm{p}+\mathrm{Ni}$ collisions The helium ions are not well visible in this representation but $\mathrm{Li}, \mathrm{Be}, \mathrm{C}$ up to $\mathrm{Si}$ lines can be distinguished, and there are also visible points in the area where $\mathrm{Al}$ and $\mathrm{Si}$ ions are expected. 
of-flight for different isotopes. The isotope separation was done by combining the information from MCPs (time-offlight) and BCD (energy deposited inside BCD volume) allowing for the separation of following isotopes: ${ }^{6} \mathrm{Li}$, ${ }^{7} \mathrm{Li},{ }^{8} \mathrm{Li}-{ }^{7} \mathrm{Be},{ }^{9} \mathrm{Be},{ }^{10} \mathrm{Be}-{ }^{10} \mathrm{~B},{ }^{11} \mathrm{~B}-{ }^{11} \mathrm{C},{ }^{12} \mathrm{C},{ }^{13} \mathrm{C},{ }^{14} \mathrm{C}$ and ${ }^{13} \mathrm{~N}$, ${ }^{14} \mathrm{~N}[22]$.

\subsection{Kinetic energy spectra using Si-detectors and comparison to model calculations}

In order to compare the measured energy spectra, we have used the INCL4.2 intra-nuclear cascade model [25] for the description of the first stage of the reaction, and the Generalized Evaporation Model GEM [26] for the second stage.

The analysis of the data with GEM coupled to the INCL4.2 model confirmed that for the $2.5 \mathrm{GeV} p+\mathrm{Au}$ reaction the low-energy component of the emitted ${ }^{4} \mathrm{He}$ (see Fig. 2) can be well reproduced by the process associated with evaporation, however, a significant contribution corresponding to the non-evaporative mechanism present in the experimental data (filled symbols in Fig. 2) in the high-energy part of the spectrum can not be accounted for in the model. Composite particles can not be produced during the fast pre-equilibrium phase modeled by the INCL4.2. The INCL program takes into account only non equilibrium processes of the first step of the reaction which are accompanied by emission of protons, neutrons and pions. As calculated by the Monte-Carlo model INCL4.2 the small forward velocity (in respect to the beam direction) of this evaporative like "moving source" is of the order of $\beta \approx 0.0036$ on average and is in agreement with the fits resulting from the phenomenological approach [22] (two moving source fit, not discussed here).

The disagreement of the energy spectra at high energies between model predictions and experiment increases for smaller emission angles, simply because the relative contribution of particles emitted prior to equilibrium

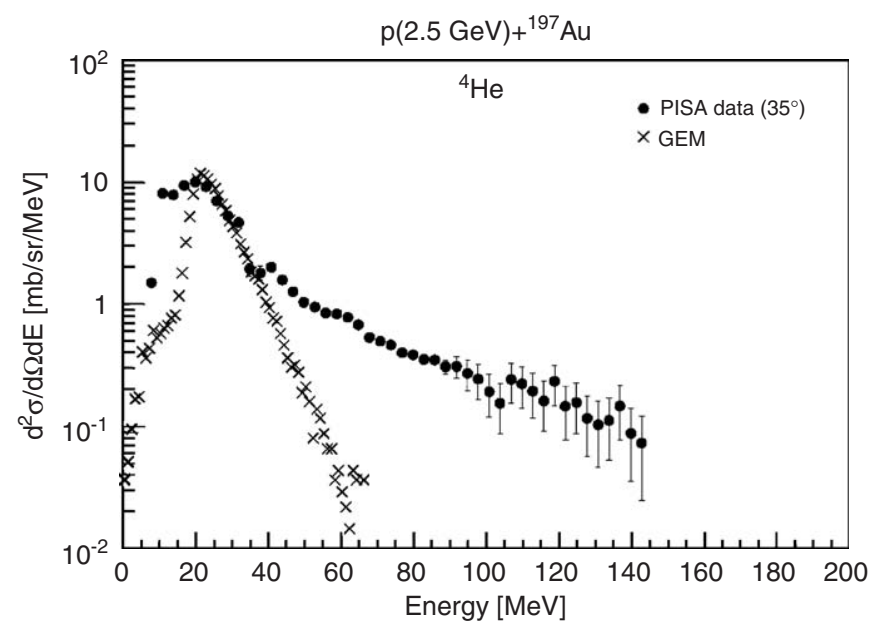

Fig. 2. Comparison between energy spectra of ${ }^{4} \mathrm{He}$ measured at $35^{\circ}$ of PISA and INCL4. 2+ GEM model calculations. increases in more forward directions. The calculations were done also for heavier particles such as $\mathrm{C}$ and $\mathrm{N}$. The comparison of the data and the model results leads to similar picture for all studied ejected particles, except that the relative ratios of the respective evaporation and preequilibrium contributions are quite different for different isotopes. Most astonishing is the fact that, even for elements as heavy as $\mathrm{C}, \mathrm{N}$ and $\mathrm{O}$, a pre-equilibrium contribution is observed experimentally which again is reflected in the spectra as calculated by the model. Actually there are currently no models on the market which can quantitatively describe the emission of composite, energetic particles in a coherent way. Here for the comparison the standard parameter set of the INCL4.2 and GEM has been applied respectively and no adjustment of any model parameter has been performed. The models allow the comparison of cross-sections on an absolute level.

\section{Conclusion}

The PISA experiment at COSY Juelich has been consulted to validate models with regard to reaction cross-sections or reaction probabilities, charged particle production cross-sections and angular- and energy-distributions following $\mathrm{GeV}$ proton induced reactions on thin $\mathrm{C}-, \mathrm{Ni}-$ and Au-targets. In summary, the experiment has shown that we are able to measure using the proposed technique the products of proton-nucleus collisions with $Z$-identification up to at least $Z=16$ and isotope identification to masses up to $13-14$ with a particularly low-energy threshold of less than $1 \mathrm{MeV} / \mathrm{A}$. The analysis of IMF particles in coincidence to highly energetic $\mathrm{H}$ - and $\mathrm{He}-$ isotopes is in progress.

\section{Acknowledgment}

We acknowledge the support of the European Community-Research Infrastructure Activity under FP6 "Structuring the European Research Area" programme (CARE, Contract No. RII3-CT-2003-506395). The project is supported by the DLR/BMBF-grant POL 01/090 and the EU-programme LIFE (Light Ion Facility Europe) as well as by the FP6-EU IP EUROTRANS under contract 516520 .

\section{References}

[1] M. Enke, et al., Nucl. Phys. A 657 (1999) 317.

[2] D. Filges, F. Goldenbaum, Y. Yariv (Eds.), (SARE-5), ISSN 1433559X, ESS 112-01-T, OECD-Headquarters, Paris, France, July 17-18, 2000, (edit.2001).

[3] C.M. Herbach, et al., in: Proceedings of the International Conference on Nuclear Data for Science and Technology ND2001, Tsukuba, Japan, NDC, JAERI, 319-1195, Japan, October 7-12, 2001.

[4] B. Appleton, in: Proceedings of ICANS-XIII Report PSI 95-02, 814, 1995.

[5] S. Nagamiya, JAERI-KEK joint project on high intensity, proton accelerator, in: nineth International Conference on radiation shield- 
ing, October 17-22nd, Tsukuba, International Congress Center, Japan, 1999.

[6] Spallation Neutron Source SNS, Status Report, Oak Ridge National Laboratory, USA; http://www.ornl.gov/sns/.

[7] The ESS Project, vol. III, TR, ISBN 3-89336-303-3, 2002.

[8] F. Goldenbaum, D. Filges: MESON2002, in: seventh International Workshop Cracow, Poland, 24-28 May 2002, World Scientific, Singapore 2002, pp. 269-280, ISBN 981-238-160-0 (2002).

[9] H. Reeves, Rev. Mod. Phys. 66 (1994) 193.

[10] R. Silverberg, C.H. Tsao, Phys. Rep. 191 (1990) 351.

[11] A.S. Botvina, D.H.E. Gross, Phys. Lett. B 344 (1995) 6.

[12] L.G. Moretto, et al., Phys. Rev. 74 (1995) 1530.

[13] J. Pochodzalla, et al., Phys. Rev. Lett. 75 (1995) 1040.
[14] The PISA Coll., IKP/COSY Annual Report 1999, Jül-3744, ISSN0944-2952, 1999, p. 175ff.

[15] Barna, et al., Nucl. Instr. and Meth. A 519 (2003) 610.

[16] R.J. McDonald, et al., Nucl. Instr. and Meth. 219 (1984) 508.

[17] A. Moroni, et al., Nucl. Instr. and Meth. 225 (1984) 57.

[18] G.D. Westfall, et al., Nucl. Instr. and Meth. A 238 (1985) 347.

[19] R. Kotte, et al., Nucl. Instr. and Meth. A 257 (1987) 244.

[20] A.A. Kotov, et al., Exp. Tech. Phys. 36 (6) (1988) 513.

[21] H. Ochiishi, et al., Nucl. Instr. and Meth 369 (1996) 269.

[22] A. Bubak, PhD-Thesis, Jül-4138, ISBN 0944-2952, 2004.

[23] A. Budzanowski, et al., IKP/COSY Annual Report 1999, p. 176.

[24] IKP Ann. Rep, Juel-3978, ISSN0944-2952, p. $210 f f, 2001$.

[25] J. Cugnon, et al., Nucl. Phys. A 620 (1997) 475.

[26] S. Furihata, Nucl. Instr. and Meth. B 171 (2000) 251. 